\section{P10.21 A STUDY ON THE USE OF IMIQUIMOD FOR THE TREATMENT OF GENITAL MOLLUSCUM CONTAGIOSUM AND GENITAL WARTS IN FEMALE PATIENTS}

${ }^{1}$ Puri Neerja*, ${ }^{2}$ Talwar Ashutosh. ${ }^{1}$ Dermatologist, Punjab Health Systems Corporation; ${ }^{2}$ Surgeon, PHSC

10.1136/sextrans-2015-052270.449

Introduction The clinical effect of imiquimod stems from cytokine-induced activation of the immune system. A randomised study was conducted to study the efficacy and safety of daily applications of $5 \%$ imiquimod cream in female patients with external genital warts and MC.

Methods Patients were randomised to receive daily applications of $5 \%$ imiquimod cream for a maximum of 16 weeks. Before bedtime patients rubbed the study cream into clean, dry, lesional skin until it disappeared and washed the area with soap and water $8 \pm 2 \mathrm{~h}$ after application. To investigate wart and $\mathrm{MC}$ recurrence, patients who had complete clearance of their baseline lesions at any time during the treatment period stopped treatment and entered a 12-week treatment free follow-up period. Patients were evaluated weekly for the first 4 weeks and every 2 weeks thereafter for the remainder of the 16-week treatment period as well as during the 12-week follow-up period.

Results The clearance rate of lesions was $75 \%$ in genital MC patients and $50 \%$ in patients with genital warts. Erythema was the commonest adverse reaction seen $24 \%$ patients with the use of $5 \%$ imiquimod. Other side effects were excoriation seen in $16 \%$ patients, erosions in $10 \%$ patients, excoriation in $6 \%$ patients and pain was seen in $4 \%$ patients.

Conclusions Nonspecific inflammation and dermatitis can occur during use of imiquimod for genital warts and molluscum. Fortunately, after completion of the therapy, the skin often heals with barely any scarring.

Disclosure of interest Nil.

\section{P10.22 ENGINEERING HUMAN RHINOVIRUS SEROTYPE-A1 AS A VACCINE VECTOR}

${ }^{1} \mathrm{~K}$ Tomusange*, 'W Yu, ${ }^{2} \mathrm{~A}$ Suhrbier, ${ }^{1} \mathrm{D}$ Wijesundara, ${ }^{1} \mathrm{~B}$ Grubor, ${ }^{1} \mathrm{E}$ Gowans. 'Virology Laboratory, Basil Hetzel Institute, Discipline of Surgery, University of Adelaide, Adelaide, South Australia, Australia; ${ }^{2}$ QIMR Berghofer Medical Research Institute, Brisbane, Queensland, Australia

\subsection{6/sextrans-2015-052270.450}

Introduction Vaccination is the optimal long-term solution to the human immuno-defficiency virus (HIV) pandemic. A majority of new HIV infections result from mucosal transmission, highlighting the need for novel vaccines that primarily generate mucosal immunity. Like HIV, human rhinovirus serotype-A1 (HRV-A1, the common cold virus) is primarily transmitted via mucosal surfaces. This makes HRV-A1 a potential vector for mucosally targeted vaccines to generate robust protection against HIV at the vaginal and other distant mucosal surfaces, and systemically.

Methods Using recombinant technology, we inserted discrete overlapping fragments of HIV gag and full-length tat into the junction between genes that encode HRV-A1 structural and nonstructural proteins to generate recombinant HRV-A1s (rHRVA1s) encoding HIV Gag and Tat proteins.

Results Transfection of H1-HeLa cells with rHRV-A1s transcripts yielded infectious and replication-competent rHRV-A1s with similar growth characteristics as wildtype HRV-A1. We also confirmed that cells infected with rHRV-A1s stably expressed Gag and Tat (beyond 5 passages), of the correct sizes and mainly localised in the cytoplasm as revealed by western blot assay, reverse-transcription polymerase chain reaction and immunofluorescence. These results have been recently accepted for publication in Virus Research Journal (reference number: VIRUS96578, April 2015).

Conclusion To the best of our knowledge, this is the first time replication-competent and stable HRV-A1 vectors have been generated. The individual rHRV-A1gag/tat generated in this study have been mixed into a cocktail vaccine and administered intranasally to female $\mathrm{Balb} / \mathrm{C}$ mice to evaluate its immunogenicity (animal experiments currently on-going). The protective efficacy of the resultant HIV-Gag-specific cell-mediated and Tat humoral responses will be documented in mice challenged intravaginally with chimeric rodent ecotropic murine leukaemia virus (EcoHIV). EcoHIV was developed by replacing the coding region of glycoprotein-120 (gp-120) of HIV strain NL4-3 with gp80 of EcoHIV to ensure that the chimeric virus infected murine cells only.

Disclosure of interest statement This work was supported by a grant from The Hospital Research Foundation (THRF), Woodville, South Australia and grant number APP1026293 from the National Health and Medical Research Council (NHMRC) of Australia. TK is a post-graduate Fellow supported by THRF while SA and GE are Senior Research Fellows of the NHMRC. The authors declare no financial or commercial conflict of interest.

\section{P11 - Sexual behaviour and STI in men who have sex with men and transgender people}

\section{P11.01 SAME-SEX BEHAVIOUR AND EXPERIENCE MEASURED ON MULTIPLE OCCASIONS IN A BIRTH COHORT REVEALS HIGHER LIFETIME PREVALENCE THAN WOULD BE FOUND IN A CROSS-SECTIONAL STUDY}

${ }^{1} \mathrm{NP}$ Dickson*, ${ }^{1} \mathrm{~K}$ Magner, ${ }^{1} \mathrm{AA}$ Righarts, ${ }^{2} \mathrm{P}$ Saxton, ${ }^{1} \mathrm{~T}$ van Roode, ${ }^{1} \mathrm{~J}$ Connor. 'Department of Preventive and Social Medicine, University of Otago, Dunedin, New Zealand; ' ${ }^{2}$ Gay Men's Sexual Health Research Group, Department of Social and Community Health, University of Auckland, New Zealand

\section{$10.1136 /$ sextrans-2015-052270.451}

Background Understanding sexual - including same-sex - behaviour is critical for appropriate policies to promote sexual health. While most information on current and past same-sex behaviour (SSB) is obtained from cross-sectional studies, the validity of information from these is not known. We have explored this in a cohort study in which questions on SSB were asked on multiple occasions over a prolonged age range.

Methods In the Dunedin Multidisciplinary Health and Development Study computer-presented questions on ever (and in the past year) having a same-sex partner (SSP), male anal intercourse (for men), and a same-sex experience (SSE) ("any contact you felt was sexual"), were asked of men on four occasions between ages 21-38, and of women on three occasions between 26-38. We have compared reports of lifetime SSP and SSE at age 38, with the summation of reports on all occasions.

Results Among men, at age 38, 12.4\% reported ever a SSP, $5.2 \%$ ever male anal intercourse, and $14.9 \%$ ever a SSE. Based on responses from all the assessments, the respective proportions reporting these behaviours were $16.9 \%, 6.5 \%$ and $21.2 \%$, 
increases of $36 \%, 25 \%$ and $42 \%$ respectively. Among the women, at age $38,16.8 \%$ reported ever having a SSP, and $22.7 \%$ ever a SSE. Based on responses from all assessments, the respective proportions were $20.5 \%$ and $31.4 \%$, increases of $22 \%$ and $45 \%$ respectively.

Conclusions In this cohort SSB was more common that reported from other population-based studies. There are three plausible explanations for the marked discrepancies between the lifetime SSB reported at age 38 and derived from all reports: (1) some reports at early assessments were erroneous, (2) with age participants were less willing to report past same-sex behaviour, (3) with age earlier SSB were forgotten or reinterpreted as not being sexual. The latter seems most plausible.

Disclosure of interest statement None of the authors have conflicts of interests to disclose.

\section{P11.02 REPRESENTING MEN WHO HAVE SEX WITH MEN (MSM) IN BRITAIN: EVIDENCE FROM COMPARATIVE ANALYSES OF THE LATEST CONVENIENCE AND PROBABILITY SURVEYS}

${ }^{1} \mathrm{P}$ Prah*, ${ }^{2} \mathrm{~F}$ Hickson, ${ }^{1} \mathrm{C}$ Bonell, ${ }^{3} \mathrm{~L}$ McDaid, ${ }^{1,2} \mathrm{~B}$ Erens, ${ }^{3}$ J Riddell, ${ }^{1} \mathrm{~S}$ Wayal, ${ }^{4} \mathrm{~A}$ Nardone, ${ }^{1} \mathrm{P}$ Sonnenberg, ${ }^{1} \mathrm{AM}$ Johnson, ${ }^{1} \mathrm{CH}$ Mercer. ${ }^{1}$ University College London; ${ }^{2}$ London School of Hygiene and Tropical Medicine; ${ }^{3}$ University of Glasgow; ${ }^{4}$ Public Health UK

\subsection{6/sextrans-2015-052270.452}

Introduction Convenience samples of MSM are typically skewed towards gay-identified and community-attached men who do not necessarily represent all MSM, a key population for STI/HIV transmission. Comparing convenience samples with a probability sample of MSM enables us to assess their representativeness.

Methods We compared 148 MSM in Britain's third National Survey of Sexual Attitudes and Lifestyles (Natsal-3), with men in Britain's key convenience surveys of gay men: 15,500 in the European MSM Internet Survey (EMIS), 1,012 in the Gay Men's Sexual Health Survey London (GMSHS-London), 1,234 in the Gay Men's Sexual Health Survey Scotland (GMSHS-Scotland), all undertaken 2010-2012. Men were aged 18-64, resident in Britain, and reported $>=1$ male sexual partners (past year). Comparisons include demographic, health characteristics and sexual behaviours, using identically-worded questions. Multivariable analyses accounted for socio-demographic differences between the samples.

Results Convenience samples had significantly younger and better educated men than Natsal-3, and a larger proportion of men identifying as gay (88\%-95\% vs. 62\%). Partner numbers were larger and same-sex anal sex more commonly reported by convenience samples but no significant differences were observed in age at first sex or reporting unprotected anal intercourse. Compared to Natsal-3, men in convenience samples were more likely to report HIV testing (past year) (all adjusted odds ratios (AORs) $>=2.30,95 \%$ CI lower bounds $>=1.49$ ) and gonorrhoea diagnoses (all AORs $>=7.99$, 95\% CI lower bounds $>=1.11$ ). However, fewer differences between samples were observed when analyses focused on MSM who identified as gay.

Conclusion Participation bias may mean convenience samples of MSM demographically misrepresent and over-estimate sexual activity and HIV testing for the entire MSM population. However, they may be more representative of gay-identified MSM, capture a broader range of behaviours and achieve larger samples, relative to general population surveys. Methods are needed to triangulate data from different surveys to strengthen the evidence-base for MSM.
P11.03 LUBRICANT USE DURING RECEPTIVE ANAL INTERCOURSE AND RECTAL CHLAMYDIAL AND GONOCOCCAL INFECTIONS AMONG MEN WHO HAVE SEX WITH MEN

${ }^{1} \mathrm{C}$ Maierhofer* ${ }^{*}{ }^{1} \mathrm{CE}$ Rice, ${ }^{2} \mathrm{KS}$ Fields, ${ }^{2} \mathrm{M}$ Ervin, ${ }^{3} \mathrm{AN}$ Turner. ${ }^{1}$ Division of Epidemiology, College of Public Health, The Ohio State University, Columbus, OH, USA; ${ }^{2}$ Sexual Health Clinic, Columbus Public Health, Columbus, OH, USA; ${ }^{3}$ Division of Infectious Diseases, Department of Internal Medicine, College of Medicine, The Ohio State University, Columbus, $\mathrm{OH}$, USA

\subsection{6/sextrans-2015-052270.453}

Introduction Despite the frequent use of lubricants during anal sex, few studies have examined associations between specific lubricant types and rectal STIs.

Methods Between July 2012 and October 2013, we conducted a cross-sectional study of men who have sex with men (MSM) recruited from an urban STD clinic in the Midwestern United States. In a self-administered survey, participants identified the types and frequency of lubricants used during receptive anal intercourse (RAI) in the previous three months. Rectal chlamydial and gonococcal infection was diagnosed using nucleic acid amplification testing. Among men who report any RAI in the previous three months, we used multivariable logistic regression models to analyse associations between recent use of nine specific lubricants and prevalent rectal infection (chlamydia or gonorrhoea).

Results Of 235 MSM in the study, $77 \%(n=182)$ reported RAI in the previous three months, and $95 \%$ of those $(\mathrm{n}=173)$ reported lubricant use during RAI during the same time period. In separate, unadjusted models, Gun Oil (OR: 2.11, 95\% CI: 1.04, 4.25), Slick (OR: 4.35, 95\% CI: 1.27, 14.89), baby oil (OR: 4.35, 95\% CI: 1.27, 14.89), Vaseline (OR: 2.53, 95\% CI: 1.08, 5.91), and precum (OR: 2.74, 95\% CI: $1.25,6.01)$ were each associated with prevalent rectal chlamydia or gonorrhoea. After adjustment for age, condom use, number of partners, and HIV status, only precum remained significantly associated with prevalent rectal infection (AOR: 3.10, 95\% CI: 1.15, 8.38).

Conclusion Rectal lubricant is a very common exposure among MSM, a population with high prevalence of STIs and HIV. In unadjusted analyses, we observed significant associations between several lubricants and rectal infection, however after multivariable adjustment, only precum was associated with prevalent infection. Longitudinal studies are needed to examine any causal relationship between specific lubricants and STI acquisition.

Disclosure of interest statement No authors have any conflict to disclose.

\section{P11.05 PROPORTION OF HIV AND SEXUALLY TRANSMITTED INFECTION (STI) CASES AMONG MEN WHO HAVE SEX WITH MEN (MSM) ATTRIBUTABLE TO SEROSORTING}

${ }^{1} \mathrm{CM}$ Khosropour* ${ }^{*},{ }^{1,2}$ LA Barbee, ${ }^{1,2} \mathrm{JC}$ Dombrowski, ${ }^{1,2}$ RP Kerani, ${ }^{1,2}$ DA Katz, ${ }^{3} \mathrm{~F}$ Swanson, $1,2 \mathrm{MR}$ Golden. 'University of Washington; ${ }^{2}$ Public Health - Seattle \& King County; ${ }^{3}$ Gay City Health Project

\subsection{6/sextrans-2015-052270.454}

Introduction The contribution of serosorting to HIV/STI disease burden among MSM is unknown.

Methods The study used data collected as part of routine care at an STD clinic 2002-2013 or a community-based HIV/STD testing centre 2004-2013 in Seattle, Washington. Data included men's condom use for anal sex with HIV-positive, negative and 